OPEN ACCESS

Edited by:

Heike Wulff,

University of California, Davis,

United States

Reviewed by:

Nishant K. Mishra,

Tulane University, United States

Yuchuan Ding,

Wayne State University School of

Medicine, United States

*Correspondence:

Joshua D. Bernstock

bernstockjd@ninds.nih.gov

tThese authors jointly supervised this work.

Specialty section: This article was submitted to Stroke, a section of the journal

Frontiers in Neurology

Received: 27 October 2017 Accepted: 20 December 2017

Published: 12 January 2018

Citation:

Bernstock JD, Ye DG, Griffin A, Lee Y-j, Lynch J, Latour LL, Friedman GK, Maric D and Hallenbeck JM (2018) Cerebral Ischemia Increases Small Ubiquitin-Like Modifier Conjugation within Human Penumbral

Tissue: Radiological-

Pathological Correlation.

Front. Neurol. 8:738.

doi: 10.3389/fneur.2017.00738

\section{Cerebral Ischemia Increases Small Ubiquitin-Like Modifier Conjugation within Human Penumbral Tissue: Radiological-Pathological Correlation}

\author{
Joshua D. Bernstock ${ }^{1,2 *}$, Daniel G. Ye', Allison Griffin ${ }^{3,4}$, Yang-ja Lee', John Lynch ${ }^{3}$, \\ Lawrence L. Latour ${ }^{3}$, Gregory K. Friedman ${ }^{5}$, Dragan Maric ${ }^{6 \dagger}$ and John M. Hallenbeck ${ }^{1 \dagger}$ \\ 'Stroke Branch, National Institute of Neurological Disorders and Stroke, National Institutes of Health, Bethesda, MD, United \\ States, ${ }^{2}$ Department of Clinical Neurosciences, Wellcome Trust-Medical Research Council Stem Cell Institute, University of \\ Cambridge, Cambridge, United Kingdom, ${ }^{3}$ Section on Stroke Diagnostics and Therapeutics, National Institute of \\ Neurological Disorders and Stroke, National Institutes of Health, Bethesda, MD, United States, ${ }^{4}$ Center for Neuroscience \\ and Regenerative Medicine at the Uniformed Services University of the Health Sciences, Bethesda, MD, United States, \\ ${ }^{5}$ Division of Pediatric Hematology and Oncology, Department of Pediatrics, University of Alabama at Birmingham, \\ Birmingham, AL, United States, ${ }^{6}$ Flow and Imaging Cytometry Core Facility, National Institute of Neurological \\ Disorders and Stroke, National Institutes of Health, Bethesda, MD, United States
}

Posttranslational modification by small ubiquitin-like modifier (SUMO) regulates myriad physiological processes within cells and has been demonstrated to be highly activated in murine brains after cerebral ischemia. Numerous in vitro and murine in vivo studies have demonstrated that this increased SUMO conjugation is an endogenous neuroprotective stress response that has potential in being leveraged to develop novel therapies for ischemic stroke. However, SUMO activation has not yet been studied in poststroke human brains, presenting a clear limitation in translating experimental successes in murine models to human patients. Accordingly, here, we present a case wherein the brain tissue of a stroke patient (procured shortly after death) was processed by multiplex immunohistochemistry to investigate SUMO activation.

Keywords: SUMOylation, ischemic stroke, neuroprotection, magnetic resonance imaging, penumbra

\section{INTRODUCTION}

An 89-year-old man presented to our facility with acute onset of left-sided weakness and hemineglect. Magnetic resonance imaging (MRI) revealed a right middle cerebral artery (MCA) stroke with associated hypoperfusion of the right MCA territory and a thrombus in the distal right internal carotid artery. The patient was treated with intravenous recombinant tissue plasminogen activator (rtPA). The patient's family provided surrogate consent, and the patient was enrolled in an IRBapproved natural history observational study (ClinicalTrials.gov NCT00009243). The patient worsened neurologically over the following $24 \mathrm{~h}$ and was placed on comfort care. The patient expired $43 \mathrm{~h}$ after hospital admission due to cardiorespiratory failure. The family provided written consent for an unrestricted donation for diagnostic, scientific, or therapeutic purposes, and an autopsy was performed within $12 \mathrm{~h}$ of death. Brain tissue was obtained at autopsy and stored at the National Cancer Institute Laboratory of Pathology. The surfaces of the cerebrum and brainstem showed mild atrophy, 
but no softening or masses. There was no evidence of herniation of the cingulate gyri, unci, or cerebellar tonsils. Postmortem MRI of the brain was obtained after fixation at 7T and co-registered to the in vivo MR images obtained at stroke onset. Brain samples for immunofluorescent staining were derived from the right frontal lobe at the periphery of the ischemic region within an area of hypoperfusion as confirmed by MRI, as well as from the contralateral region of the left frontal lobe; the penumbra was characterized as areas visualized as normal for diffusion but abnormal for perfusion (Figure 1).

\section{BACKGROUND AND DISCUSSION}

Posttranslational modification by small ubiquitin-like modifier (SUMO) regulates diverse homeostatic processes within cells (1). After the demonstration of SUMO activation during hibernation torpor (2), further experiments have shown that both global and focal transient cerebral ischemia-reperfusion dramatically increase the levels and nuclear localization of SUMO-conjugated proteins within murine brains, and furthermore, this process may contribute toward neuroprotection $(3-9,19)$. Consequently, numerous studies in murine models have sought to induce protection against ischemia by leveraging SUMOylation (10-12). However, at present, evidence of SUMO activation in postischemic human brain tissue has yet to be demonstrated.

Herein, fluorescent multiplex immunohistochemistry (mIHC) was employed to visualize the intensity and localization of SUMO within the neurons of the penumbral tissue and the corresponding tissue of the contralateral hemisphere. Upregulation of SUMO1 and SUMO2/3 in neurons of the ischemic penumbra was clearly observed in the form of an increased intensity of immunoreactivity compared to the matched contralateral tissue (Figure 2). Furthermore, SUMO1 and SUMO2/3 immunoreactivity was also observed to translocate from the cytoplasm to the nucleus (visualized with DAPI) in greater intensity in penumbral neurons compared to contralateral neurons (Figure 3).

Briefly, after an ischemic event, the affected tissue can be divided into three regions based on factors including collateral perfusion, susceptibility to cell death, and duration of blood vessel occlusion: the ischemic core, the ischemic penumbra, and the oligemia (13-16). The ischemic core is tissue that has suffered irreversible injury due to severe reductions in blood flow $(<20 \%$ of normal) and oxygen delivery and is as such considered unsalvageable $(15,17)$. The ischemic penumbra comprises severely hypoperfused ( $40 \%$ of normal) tissue, is functionally impaired, is progressively recruited into the ischemic core as the duration of vascular occlusion increases (being detectable with PET as late as $18 \mathrm{~h}$ after onset), and is considered salvageable if perfusion is restored $(15,17,18)$. Thus, the ischemic penumbra is the target of acute reperfusion therapies (e.g., thrombolysis using rtPA), which improve patient outcomes in proportion to the volume of penumbra ultimately salvaged (18). Finally, the oligemia surrounds the penumbra and suffers a mildly reduced CBF ( $>40 \%$ of normal). Tissue in the oligemia is generally not at risk barring complications (15). Ultimately, as the ischemic penumbra is where therapeutic interventions in ischemic stroke are most relevant and efficacious (with the understanding that the SUMO pathway has been identified as a potential therapeutic target in animal models of stroke), and considering that SUMOylation in the brain is a rapidly cycling ATP-dependent process $(1,8)$, tissue from the ischemic penumbra was selected for mIHC.

Past studies in murine models of ischemia have demonstrated increased SUMO conjugation in the brain after ischemiareperfusion. Notably, the greatest levels of SUMO activation and neuronal nuclear localization of SUMO2/3-conjugated proteins were observed in cells of the ischemic penumbra $(4,19)$. Significant research investigating the neuroprotective effect of SUMO activation in in vitro and murine models of ischemia have already been conducted, demonstrating the critical role of SUMO activation as an endogenous neuroprotective stress response that attenuates neuronal damage caused by ischemia-reperfusion, as well as enhanced neuroprotection when SUMO activation is induced above physiological levels $(2,5-7,10,20-22)$. Cells overexpressing the SUMO E2 conjugase, Ubc9, and subsequently demonstrating increased SUMO conjugation, were protected from oxygen/glucose deprivation (OGD)-induced damage, whereas cells with an inhibited Ubc9 demonstrated decreased SUMO conjugation and were sensitized to OGD-induced damage (2). The overexpression of SUMO1 and SUMO2 protected cells from OGD-induced damage, while microRNA-induced depletion of endogenous SUMO1 and SUMO2/3 sensitized cells to OGD-induced damage $(5,20)$. Suppressing SUMOylation through overexpression of SENP1 also sensitized cortical neurons to OGD-induced damage (21). Transgenic mice overexpressing Ubc9 had increased levels of SUMOylated proteins in their brains, were more tolerant to ischemic stress, and had smaller infarct volumes after pMCAO compared to wild-type mice (10). Meanwhile, transgenic SUMO-knockdown mice in which miRNAs specifically silenced SUMO1-3 in neurons displayed worse functional outcomes after transient forebrain ischemia compared to wild-type mice (9).

Naturally, as upregulation of the SUMOylation pathway has been shown to be neuroprotective in preclinical models of brain ischemia, several studies have sought to pharmacologically modify SUMOylation in an effort to bring this pathway to bear on acute ischemic insults $(11,12,23)$. For example, a lead compound (N106) has been identified to increase SUMOylation via activation of the SUMO E1 enzyme and was capable of inducing protection against myocardial insults in vitro and in vivo (23). Other compounds such as histone deacetylase inhibitors and synthetic retinoids, which increase SUMOylation by inhibiting the regulatory miRNAs miR-182 and miR-183, have demonstrated protection of cortical neurons in vitro (11). After the discovery that quercetin is a potential SENP inhibitor that may induce neuroprotection in part via increased SUMOylation (12), quantitative high-throughput screens using physiologically relevant SUMO substrates have also been developed and employed to search for potential neuroprotective compounds that act through the inhibition of SENP2 (24). The topic of therapeutic strategies leveraging SUMOylation in brain ischemia has been recently reviewed in greater detail (25). Ultimately, the search for SUMOactivating compounds that may be developed into neuroprotective therapies in humans in ongoing. 

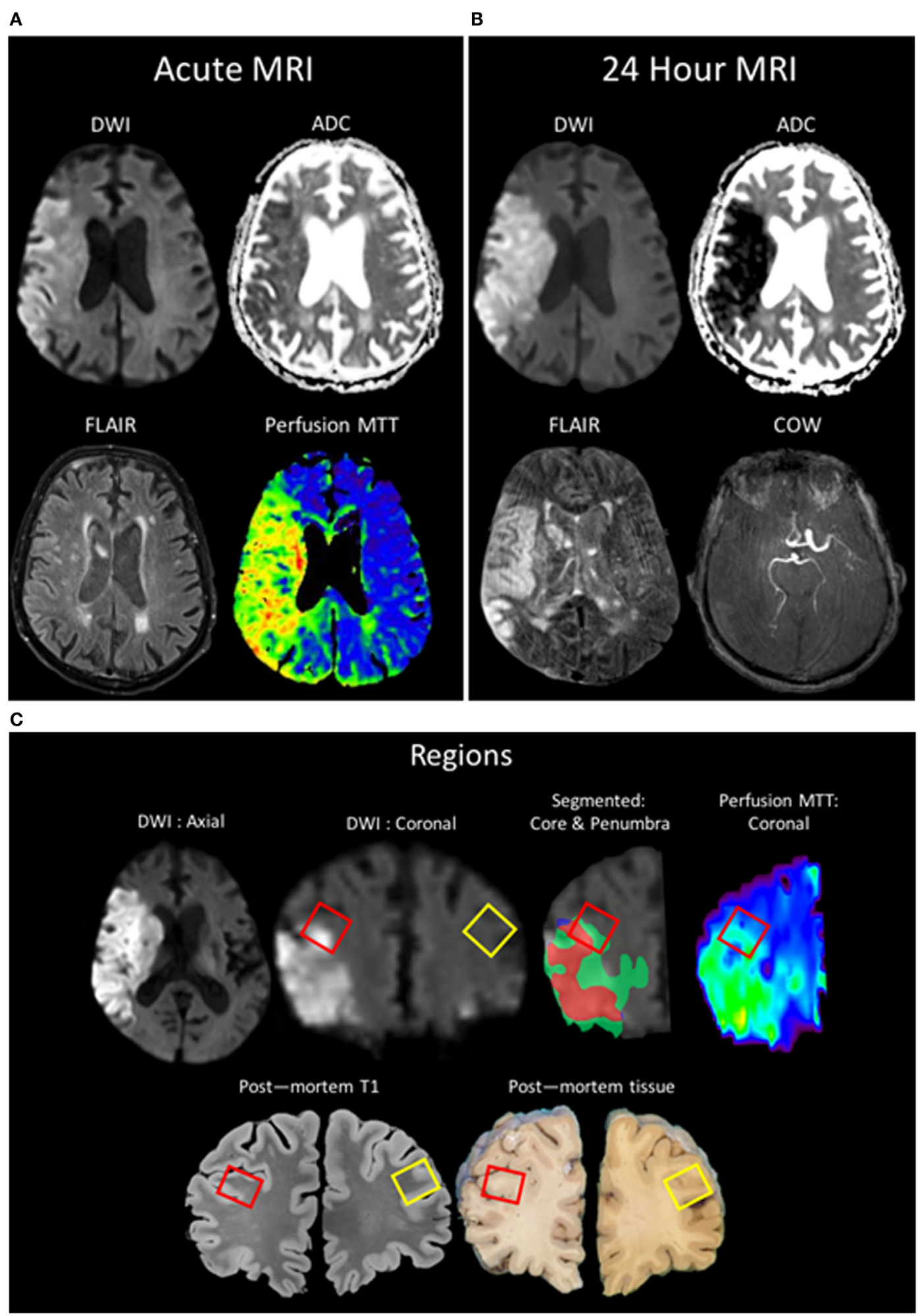

FIGURE 1 | Patient MR images taken during clinical evaluation (acute) (A) and $24 \mathrm{~h}$ later (B) [pictured: trace-weighted diffusion weighted imaging (DWI) and ADC maps, fluid-attenuated inversion recovery (FLAIR), perfusion mean-transit time (MTT), and a minimum intensity projection of the circle of willis]. (C) MR images taken postmortem were analyzed to co-localize regions of interest identified in vivo with the postmortem sample (red boxes: ipsilateral ROI; yellow boxes: contralateral). Two regions of interest were segmented, the region of the core (red) taken from the baseline DWI and the region of the penumbra as the mismatch between the MTT and the DWI (green), and overlaid onto the 24-h DWI. 

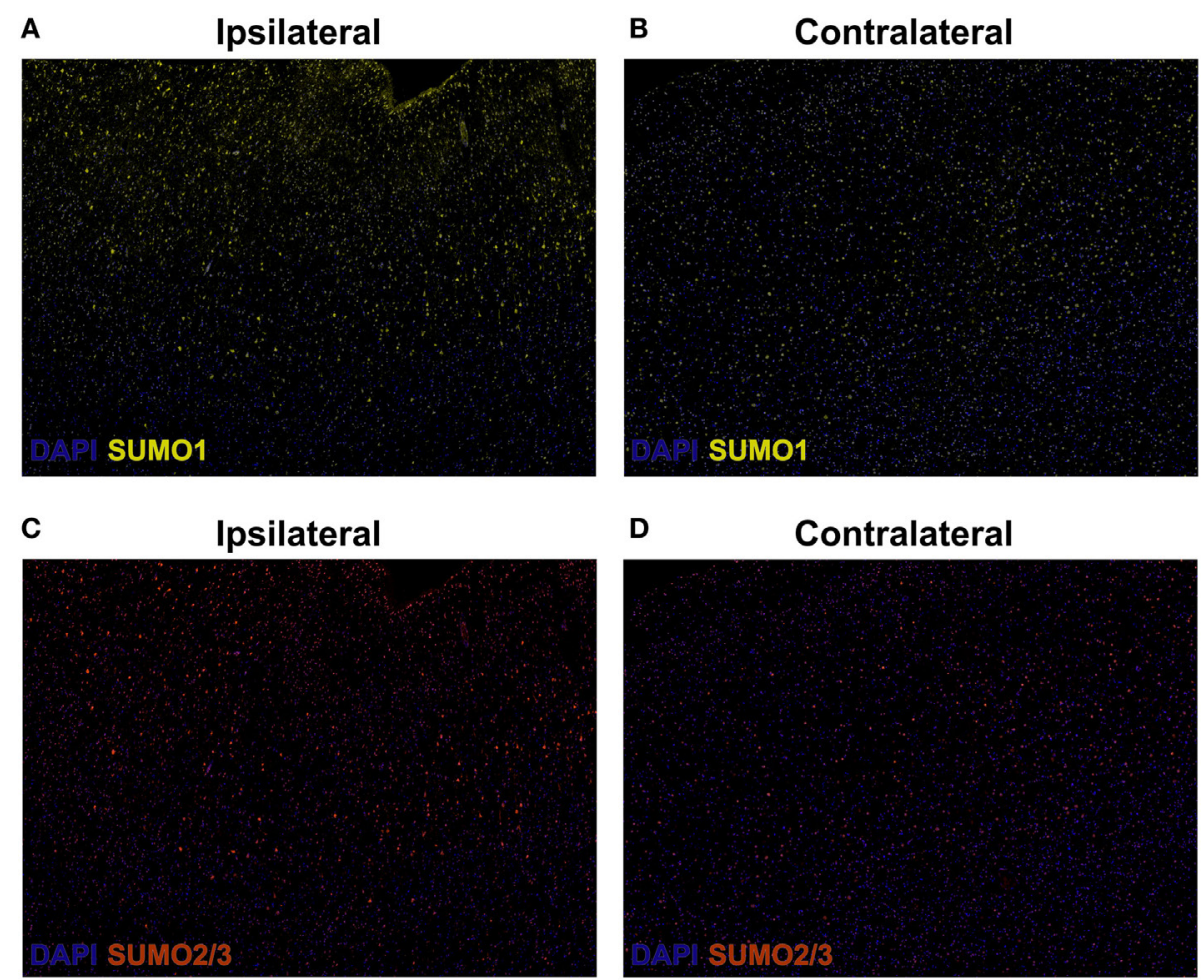

FIGURE 2 | Composite wide-field fluorescent multiplex immunohistochemistry (mIHC) images (yellow, SUMO1; red, SUMO2/3; blue, DAPI,). (A) Ipsilateral, SUMO1. (B) Contralateral, SUMO1. (C) Ipsilateral, SUMO2/3. (D) Contralateral, SUMO2/3. Capture parameters of ipsilateral and contralateral images were identical. Cropped ROls were taken from comparable layers of cortex. The intensity of SUMO1 and SUMO2/3 immunoreactivity is increased in neurons residing within the penumbral tissue compared to neurons in the matched contralateral anatomy.

\section{CONCLUDING REMARKS}

To date, despite numerous animal studies and the clinical importance of developing neuroprotective therapies for ischemic stroke, there has been no evidence demonstrating SUMO activation in human brains after stroke. This report is the first to document that within a poststroke human brain, SUMOylation patterns are largely concordant with those in postischemic murine brains, suggesting that enhanced SUMO conjugation may play a similar role in humans as in murine models (i.e., as an endogenous neuroprotective stress response that could be therapeutically leveraged to attenuate ischemic damage and promote functional recovery). Thus, it is the authors' contention that this case serves as an initial validation of the body of research into SUMO conjugation-induced neuroprotection, although it must be recognized that more studies should be conducted to confirm the generalizability of these results.

\section{MATERIALS AND METHODS}

\section{Magnetic Resonance Image Acquisition}

As a part of the baseline clinical evaluation for stroke, and research follow-up, the subject was imaged on a 3T MR Scanner (Siemens Medical, Malvern, PA, USA) using a standardized MRI protocol that included diffusion-weighted imaging
(DWI), T2* gradient recalled echo, time-of-flight magnetic resonance angiogram of the circle of willis, and T2-weighted fluid-attenuated inversion recovery (FLAIR). Relevant parameters for sequences presented are as follows: forty $3.5-\mathrm{mm}$ thick axial-oblique slices aligned along the anterior-posterior commissure were acquired co-localized for DWI and FLAIR. DWI consisted of 15 direction tensor sequences used to generate both trace-weighted DWI and ADC maps, $b$-value $=1,000$, TR/ $\mathrm{TE}=100,025 \mathrm{x} \mathrm{ms}, \mathrm{FOV}=24 \mathrm{~cm}$, and $3 \mathrm{~mm} \times 3 \mathrm{~mm} \times 7 \mathrm{~mm}$ voxels. FLAIR consisted of TR/TE/TI $=9,000 / 120 / 2,600 \mathrm{~ms}$. Dynamic susceptibility contrast perfusion-weighted imaging was performed using an echo-planar $\mathrm{T} 2^{*}$ weighed gradient recalled echo sequence with the relevant parameters: TR/ $\mathrm{TE}=1,200 / 25 \mathrm{~ms}, \mathrm{FA}=80, \mathrm{FOV}=220 \mathrm{~mm}$, matrix of $96 \times 96$, twenty 7 -mm axial-oblique slices, and 80 dynamics after a weight-adjusted single dose of Gd-BOPTA injected at $5 \mathrm{ml} / \mathrm{s}$ into the antecubital vein. Maps of mean-transit time were generated using vendor provided software with arterial input function deconvolution.

After extraction and 1-month fixation with $10 \%$ formalin, the brain was hydrated for 1 week in $1 \%$ formalin before being transferred to a custom-machined container attached to a $1 / 3$-horsepower vacuum pump. The brain was then immersed under vacuum in fluorinated oil (Fomblin LC/8, Solvay Solexis Inc.) that was free of proton MR signal. Whole brain images were 

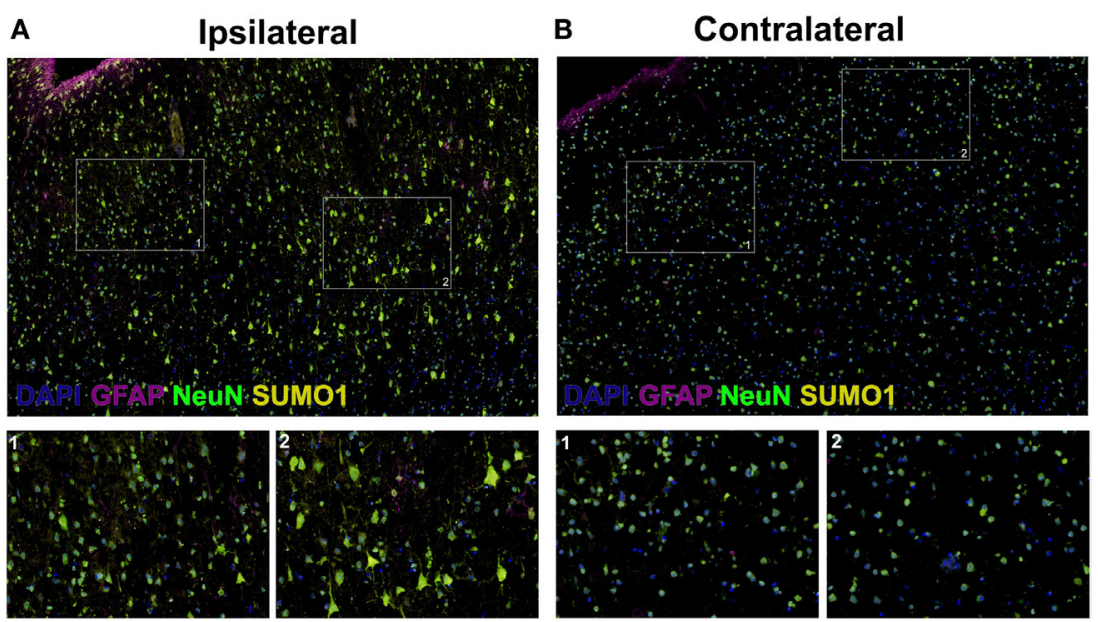

c

Ipsilateral

D

Contralateral
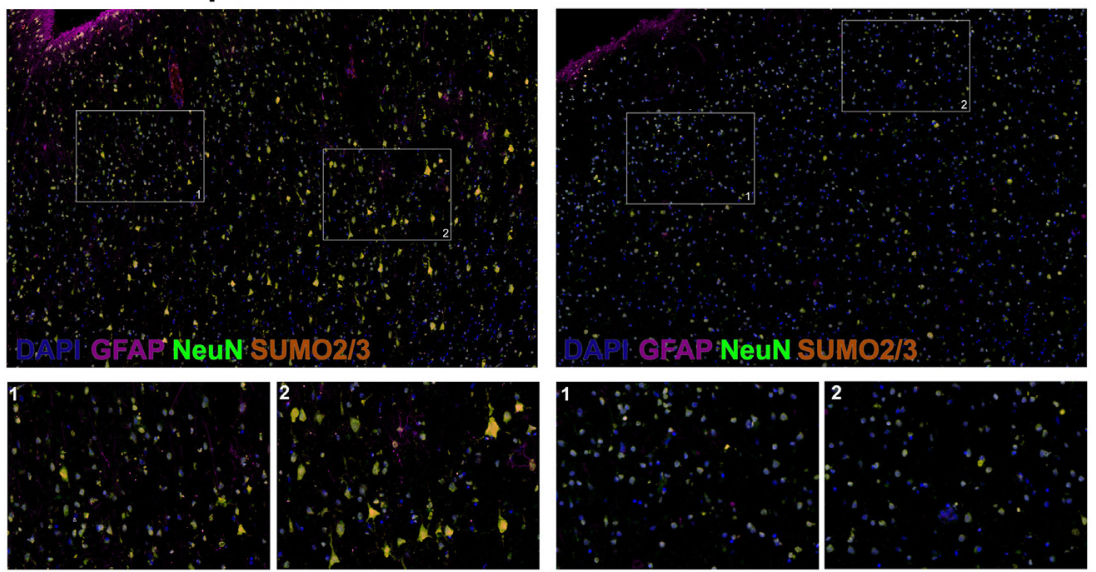

FIGURE 3 | High-resolution composite fluorescent multiplex immunohistochemistry (mIHC) images (yellow, SUMO1; red, SUMO2/3; blue, DAPI; green, NeuN; violet, GFAP). (A) Ipsilateral, SUMO1. (B) Contralateral, SUMO1. (C) Ipsilateral, SUMO2/3. (D) Contralateral, SUMO2/3. Capture parameters of ipsilateral and contralateral images were identical. Cropped ROls were taken from comparable layers of cortex. The intensity of SUMO1 and SUMO2/3 immunoreactivity is increased in neurons residing within the penumbral tissue compared to neurons in the matched contralateral anatomy; in addition, SUMO1 and SUMO2/3 immunoreactivity is translated from the cytoplasm to the nucleus in penumbral neuronal tissue.

acquired using a 7T MRI scanner (Siemens, Erlangen, Germany) and analyzed to co-localize regions of interest that had been seen in vivo to the postmortem specimen. The brain was sectioned into coronal slices, and additional sectioning and histopathological processing was performed on ROIs and matching contralateral control tissues.

\section{Fluorescence mIHC}

Briefly, $10-\mu \mathrm{m}$-thick human brain OCT-embedded sections were incubated with Human BD Fc Blocking solution (BD Biosciences) to block endogenous $\mathrm{Fc}$ receptors and then incubated in True Black Reagent (Biotium) to quench intrinsic tissue autofluorescence. The sections were then immunoreacted for $1 \mathrm{~h}$ at RT using $1-5 \mu \mathrm{g} / \mathrm{ml}$ cocktail mixture of the following immunocompatible primary antibodies: rat IgG2a anti-SUMO 1 (Sigma-Aldrich), mouse IgG1 anti-SUMO 2/3 (Abcam), mouse IgG2b anti-GFAP (BD Biosciences), and guinea pig IgG antiNeuN (EMD Millipore). This step was followed by washing off excess primary antibodies with PBS supplemented with $1 \mathrm{mg} / \mathrm{ml}$ bovine serum albumin and staining the sections using a $1 \mu \mathrm{g} / \mathrm{ml}$ cocktail mixture of the appropriately cross-adsorbed secondary antibodies conjugated to one of the following spectrally compatible fluorophores (all purchased from Thermo Fisher): Alexa Fluor 488, Alexa Fluor 546, Alexa Fluor 594, and Alexa Fluor 647. After washing off excess secondary antibodies, sections were counterstained using $1 \mu \mathrm{g} / \mathrm{ml}$ DAPI (Thermo Fisher) for visualization of cell nuclei. Slides were then coverslipped using Immu-Mount medium (Thermo Fisher) and imaged using a multichannel wide-field epifluorescence microscope (see below).

\section{Fluorescence mIHC Image Acquisition}

Images were acquired from whole specimen sections using the Axio Imager.Z2 slide scanning fluorescence microscope (Zeiss) equipped with a $20 \times / 0.8$ Plan-Apochromat (Phase-2) non-immersion objective (Zeiss), a high-resolution ORCA-Flash4.0 sCMOS 
digital camera (Hamamatsu), a 200 W X-Cite 200DC broad band lamp source (Excelitas Technologies), and five customized filter sets (Semrock) optimized to detect the following fluorophores: DAPI, Alexa Fluor 488, Alexa Fluor 546, Alexa Fluor 594, and Alexa Fluor 647. Image tiles $(600 \mu \mathrm{m} \times 600 \mu \mathrm{m}$ viewing area $)$ were individually captured at $0.325 \mu \mathrm{m}$ /pixel spatial resolution, and the tiles were seamlessly stitched into whole specimen images using the ZEN 2 image acquisition and analysis software program (Zeiss), with an appropriate color table having been applied to each image channel to either match its emission spectrum or to set a distinguishing color balance. Pseudocolored stitched images were then exported to Adobe Photoshop and overlaid as individual layers to create multicolored merged composites.

\section{ETHICS STATEMENT}

This study was performed on postmortem human brain procured after the participant's family provided written informed

\section{REFERENCES}

1. Flotho A, Melchior F. Sumoylation: a regulatory protein modification in health and disease. Ann Rev Biochem (2013) 82:357-85. doi:10.1146/AnnurevBiochem-061909-093311

2. Lee YJ, Miyake S, Wakita H, McMullen DC, Azuma Y, Auh S, et al. Protein SUMOylation is massively increased in hibernation torpor and is critical for the cytoprotection provided by ischemic preconditioning and hypothermia in SHSY5Y cells. J Cereb Blood Flow Metab (2007) 27(5):950-62. doi:10.1038/ sj.jcbfm. 9600395

3. Cimarosti H, Lindberg C, Bomholt SF, Ronn LC, Henley JM. Increased protein SUMOylation following focal cerebral ischemia. Neuropharmacology (2008) 54(2):280-9. doi:10.1016/j.neuropharm.2007.09.010

4. Yang W, Sheng H, Warner DS, Paschen W. Transient global cerebral ischemia induces a massive increase in protein sumoylation. J Cereb Blood Flow Metab (2008) 28(2):269-79. doi:10.1038/sj.jcbfm.9600523

5. Datwyler AL, Lattig-Tunnemann G, Yang W, Paschen W, Lee SL, Dirnagl $\mathrm{U}$, et al. SUMO2/3 conjugation is an endogenous neuroprotective mechanism. J Cereb Blood Flow Metab (2011) 31(11):2152-9. doi:10.1038/jcbfm. 2011.112

6. Lee YJ, Hallenbeck JM. SUMO and ischemic tolerance. Neuromolecular Med (2013) 15(4):771-81. doi:10.1007/s12017-013-8239-9

7. Lee YJ, Mou Y, Klimanis D, Bernstock JD, Hallenbeck JM. Global SUMOylation is a molecular mechanism underlying hypothermia-induced ischemic tolerance. Front Cell Neurosci (2014) 8:416. doi:10.3389/fncel.2014.00416

8. Yang W, Sheng H, Thompson JW, Zhao S, Wang L, Miao P, et al. Small ubiquitin-like modifier 3-modified proteome regulated by brain ischemia in novel small ubiquitin-like modifier transgenic mice: putative protective proteins/pathways. Stroke (2014) 45(4):1115-22. doi:10.1161/STROKEAHA. 113.004315

9. Zhang L, Liu X, Sheng H, Liu S, Li Y, Zhao JQ, et al. Neuron-specific SUMO knockdown suppresses global gene expression response and worsens functional outcome after transient forebrain ischemia in mice. Neuroscience (2017) 343:190-212. doi:10.1016/j.neuroscience.2016.11.036

10. Lee YJ, Mou Y, Maric D, Klimanis D, Auh S, Hallenbeck JM. Elevated global SUMOylation in Ubc9 transgenic mice protects their brains against focal cerebral ischemic damage. PLoS One (2011) 6(10):e25852. doi:10.1371/journal. pone. 0025852

11. Bernstock JD, Lee YJ, Peruzzotti-Jametti L, Southall N, Johnson KR, Maric D, et al. A novel quantitative high-throughput screen identifies drugs that both activate SUMO conjugation via the inhibition of microRNAs 182 and 183 and facilitate neuroprotection in a model of oxygen and glucose deprivation. J Cereb Blood Flow Metab (2016) 36(2):426-41. doi:10.1177/ 0271678X15609939 consent for an unrestricted autopsy. While living, the family gave written informed consent for the study as well as subsequent publication of medically relevant findings. The study (NCT00009243) was approved by the local Institutional Review Board.

\section{AUTHOR CONTRIBUTIONS}

All authors participated in the drafting/revision of the manuscript.

\section{FUNDING}

This work was supported by the Intramural Research Program of the NINDS/NIH and the Center for Neuroscience and Regenerative Medicine (Department of Defense). JB was also supported by an NIH-OxCam Fellowship.

12. Lee YJ, Bernstock JD, Nagaraja N, Ko B, Hallenbeck JM. Global SUMOylation facilitates the multimodal neuroprotection afforded by quercetin against the deleterious effects of oxygen/glucose deprivation and the restoration of oxygen/glucose. J Neurochem (2016) 138(1):101-16. doi:10.1111/jnc.13643

13. Astrup J, Siesjo BK, Symon L. Thresholds in cerebral ischemia - the ischemic penumbra. Stroke (1981) 12(6):723-5. doi:10.1161/01.str.12.6.723

14. Kaufmann AM, Firlik AD, Fukui MB, Wechsler LR, Jungries CA, Yonas H. Ischemic core and penumbra in human stroke. Stroke (1999) 30(1):93-9. doi:10.1161/01.STR.30.1.93

15. Baron JC. Perfusion thresholds in human cerebral ischemia: historical perspective and therapeutic implications. Cerebrovasc Dis (2001) 11(Suppl 1): 2-8. doi:10.1159/000049119

16. Hossmann KA. Cerebral ischemia: models, methods and outcomes. Neuropharmacology (2008) 55(3):257-70. doi:10.1016/j.neuropharm.2007. 12.004

17. Dirnagl U, Iadecola C, Moskowitz MA. Pathobiology of ischaemic stroke: an integrated view. Trends Neurosci (1999) 22(9):391-7. doi:10.1016/ S0166-2236(99)01401-0

18. Soares BP, Chien JD, Wintermark M. MR and CT monitoring of recanalization, reperfusion, and penumbra salvage: everything that recanalizes does not necessarily reperfuse! Stroke (2009) 40(3 Suppl):S24-7. doi:10.1161/ STROKEAHA.108.526814

19. Yang W, Sheng H, Warner DS, Paschen W. Transient focal cerebral ischemia induces a dramatic activation of small ubiquitin-like modifier conjugation. JCereb Blood Flow Metab (2008) 28(5):892-6. doi:10.1038/sj.jcbfm. 9600601

20. Lee YJ, Castri P, Bembry J, Maric D, Auh S, Hallenbeck JM. SUMOylation participates in induction of ischemic tolerance. JNeurochem (2009) 109(1):257-67. doi:10.1111/j.1471-4159.2009.05957.x

21. Cimarosti H, Ashikaga E, Jaafari N, Dearden L, Rubin P, Wilkinson KA, et al. Enhanced SUMOylation and SENP-1 protein levels following oxygen and glucose deprivation in neurones. J Cereb Blood Flow Metab (2012) 32(1):17-22. doi:10.1038/jcbfm.2011.146

22. Lee YJ, Johnson KR, Hallenbeck JM. Global protein conjugation by ubiquitin-like-modifiers during ischemic stress is regulated by microRNAs and confers robust tolerance to ischemia. PLoS One (2012) 7(10):e47787. doi:10.1371/ journal.pone.0047787

23. Kho C, Lee A, Jeong D, Oh JG, Gorski PA, Fish K, et al. Small-molecule activation of SERCA2a SUMOylation for the treatment of heart failure. Nat Commun (2015) 6:7229. doi:10.1038/ncomms8229

24. Bernstock JD, Ye D, Smith JA, Lee YJ, Gessler FA, Yasgar A, et al. Quantitative high-throughput screening identifies cytoprotective molecules that enhance SUMO conjugation via the inhibition of SUMO-specific protease (SENP)2. FASEB J (2017). doi:10.1096/fj.201700711R 
25. Bernstock JD, Yang W, Ye DG, Shen Y, Pluchino S, Lee Y-J, et al. SUMOylation in brain ischemia: patterns, targets, and translational implications. J Cerebral Blood Flow Metab (2018) 38(1):5-16. doi:10.1177/0271678x17742260

Conflict of Interest Statement: This research was conducted in the absence of any commercial or financial relationships that could be construed as a potential conflict of interest.
Copyright (® 2018 Bernstock, Ye, Griffin, Lee, Lynch, Latour, Friedman, Maric and Hallenbeck. This is an open-access article distributed under the terms of the Creative Commons Attribution License (CC BY). The use, distribution or reproduction in other forums is permitted, provided the original author(s) or licensor are credited and that the original publication in this journal is cited, in accordance with accepted academic practice. No use, distribution or reproduction is permitted which does not comply with these terms. 